\title{
Fast measurement of plasma potential, temperature and density in SOL of ASDEX Upgrade
}

\author{
J. Horacek ${ }^{1}$, J. Adamek ${ }^{1}$, H.W. Müller ${ }^{2}$, J. Seidl ${ }^{1,3}$, V. Rohde ${ }^{2}$, \\ F. Mehlmann ${ }^{4}$, C. Ionita ${ }^{4}$, A.H. Nielsen ${ }^{5}$ and ASDEX Upgrade \\ Team $^{3}$ \\ ${ }^{1}$ Institute of Plasma Physics AS CR, v.v.i., Association EURATOM/IPP.CR, Za \\ Slovankou 1782/3, 182 00, Praha 8, Czech Republic \\ ${ }^{2}$ Max Planck Institut für Plasmaphysik, EURATOM Association, Garching, \\ Germany \\ ${ }^{3}$ Institute of Theoretical Physics, Faculty of Mathematics and Physics, Charles \\ University, V Holesovickach 218000 Praha 8, Czech Republic \\ ${ }^{4}$ Association EURATOM/ÖAW, Institute for Ion Physics and Applied Physics, \\ University of Innsbruck, Austria \\ ${ }^{5}$ Association Euratom Risø DTU, Risoe National Laboratory for Sustainable \\ Energy, DK-4000 Roskilde, Denmark \\ E-mail: horacek@ipp.cas.cz
}

\begin{abstract}
This paper focuses on interpretation of fast measurement of plasma density, potential and electron temperature in SOL. Steady-state radial profiles demonstrate credibility of the Ball-pen probe. Spatial and temporal scales are found consistent with expectations based on interchange driven turbulence model generating blobs. Conditionally averaged signals found for both potential and density are also consistent, however, those for temperature show unexpected short drop at the very center of a blob.
\end{abstract}

Submitted to: Nuclear Fusion

\section{Introduction}

Simultaneous fast measurements of the plasma potential, density and electron temperature are quite a difficult task. The commonly used diagnostics suffer either from too slow or complicated electronics to recognize individual blob events (eg. fast swept Langmuir probe [1]) or low spatial resolution as using the tunnel probe [2]. In 2009, we performed experiments using the reciprocating midplane manipulator in the SOL of ASDEX Upgrade, equiped with two types of electrostatic probes. The first is a Langmuir probe, measuring either the floating potential, $V_{f l}=\phi-\alpha_{l p} T_{e}$; with $\alpha_{l p}=2.8$, (for Deuterium) or, when negatively biased, ion saturation current, $I_{s a t} \propto n_{e} \sqrt{T_{e}}$. Second, a relatively novel diagnostic, the so-called Ball-pen probe, BPP [3], measuring nearly 
Fast measurement of plasma potential, temperature and density in SOL of ASDEX Upgrade2

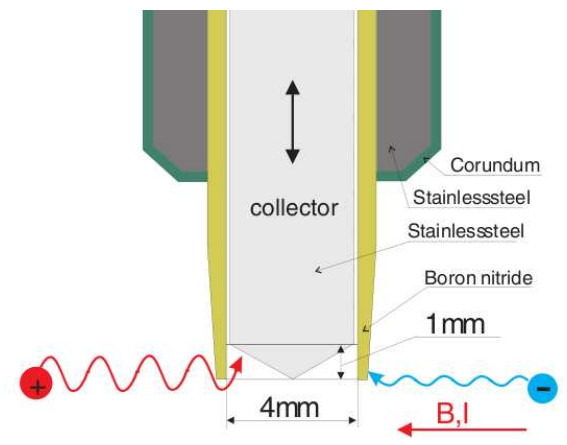

Figure 1. Schema demonstrating principle of a ball-pen probe.

plasma potential, $B P P=\phi-\alpha_{b p p} T_{e}$ with $\alpha_{b p p}$ close to zero. The difference between both signals, $\left(\phi V_{f l}\right) /\left(\alpha_{L P}-\alpha_{B P P}\right)$ yields therefore local and instantaneous values of the electron temperature, $T_{e}$. In details, the experimental arrangement is described later in Eq. (1)-Eq. (6). This concept is similar to the triple probe technique [4], with advantage of direct and fast measurement of plasma potential.

Ball-pen probe diagnostic (similar to the Katsumata probe) profits from a simple modification of the Langmuir probe in strongly magnetized plasma: the conducting collector (ie. the probe) is inserted inside a non-conductive hole to provide a magnetic shadow as depicted in Fig.1. For ion and electron currents to balance, $I^{+}+I^{-}=0$, floating Langmuir probe gets biased negatively (down to $V_{\mathrm{fl}}$ ) by plasma itself, in order to repel electrons that move faster than ions. Theoretically, $V_{f l}=\phi-\alpha \cdot T_{e}$, where $\alpha=\ln I_{\text {sat }}^{+} / I_{\text {sat }}^{-}$. This logarithmic ratio of ion to electron saturation currents is around 2.8 (for cylindrical probe in Deuterium plasma, assuming $T_{i}=T_{e}$ ). The BPP screens the electrons by magnetic field in order to achieve $\alpha \approx 0$, because ions with larger Larmor radius can penetrate inside the hole, whilst the electrons cannot. Detailed comparison with other diagnostics demonstrated such functionality [5, 3] Especially, direct comparison with $100 \mathrm{kHz}$-swept Langmuir probe $L P_{3}$ shows quite good agreement [7] in absolute values up to high frequencies. It was found experimentally [3] that $\alpha_{b p p}=0.6 \pm 0.3$. Therefore, BPP measures plasma potential with $2.8 / 0.6=5 \mathrm{x}$ smaller parasitic influence from $T_{e}$ than a single Langmuir probe. Obviously, difference between signals from a ball-pen and a Langmuir probe placed on nearby magnetic field line yields fast $T_{e}$ fluctuation measurement.

\section{Experimental setup}

The probe head was mounted on fast reciprocating manipulator located just above lowfield side midplane of the tokamak ASDEX Upgrade, as shown in Fig.2a. It contains four BPP's and four Langmuir probes $\left(L P_{1-4}\right)$ as shown in Fig.2b. The ball-pen probe collectors are inserted 1-2 mm deep inside the hole. They are made of stainless steel with diameters of $4 \mathrm{~mm}$, which are fixed inside the ceramic shielding tubes with inner 


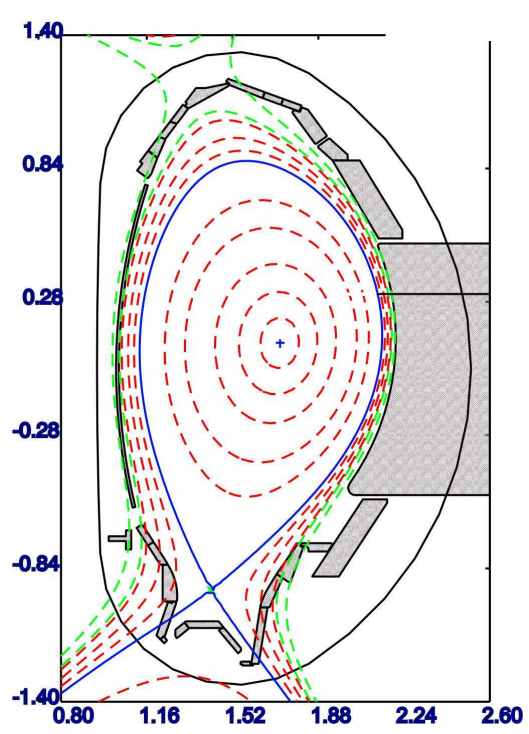

a)

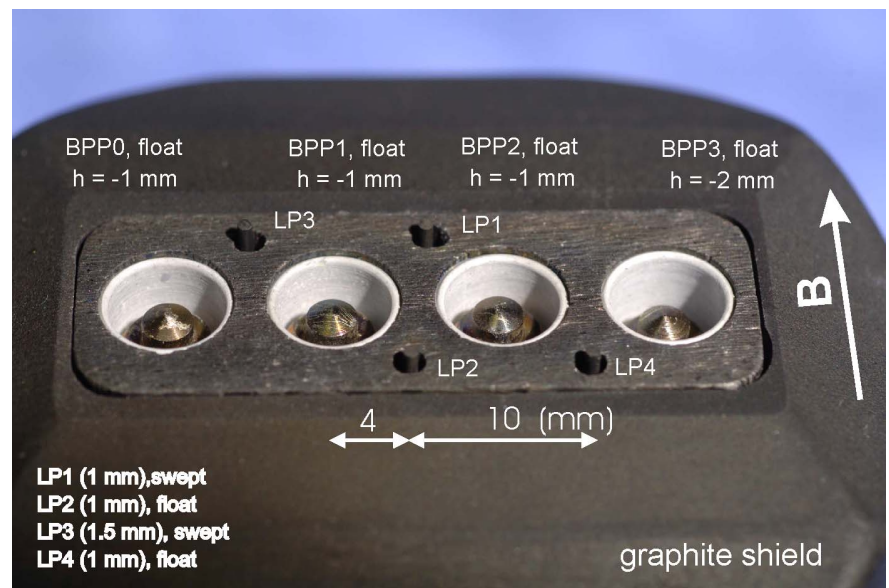

b)

Figure 2. a) Plasma geometry of tokamak ASDEX Upgrade with the reciprocating probe reaches LCFS just above LFS midplane, $\mathrm{Z}=0.28 \mathrm{~m}$. b) Photograph of the reciprocating probe head with four Langmuir pins and four Ball-pen probes. The direction parallel (poloidal) corresponds to horizontal (vertical) axis of the image. Quantities $\phi, T_{e}, n_{e}$ are measured at the depicted positions.

diameters of $6 \mathrm{~mm}$. All probes were connected as floating, except from probe $L P_{1}$ set to measure the ion saturation current. None of the probes shields another, even the pins $L P_{1}$ and $L P_{2}$ are slightly shifted poloidally and $L P_{3}$ swept to provide IVcharacteristic used in Fig.3c. The entire probe head (with diameter $60 \mathrm{~mm}$ ) is covered by a graphite protection against the high heat flux of the plasma. The time resolution of the measurements is limited only by $2 \mathrm{MHz}$ sampling frequency of the data acquisition system.

The experiments were performed in L-mode, across the SOL with a neutral beam injection (NBI) power of $1 \mathrm{MW}$, toroidal magnetic field $\mathrm{B}=2.5 \mathrm{~T}$ (1.9 T in SOL), plasma current $I_{P}=0.8 \mathrm{MA}$ and line averaged density $n_{e}=3 \cdot 10^{19} \mathrm{~m}^{3}$. The analysed data come from the second stroke of discharge 24349; its reproducibility is verified in shot 24348.

\section{Basic statistics}

We naturally define basic physics quantities in position in the middle of the head, using the experimentally obtained data (evolving in time) from probes $B P P_{1,2}$ and $L P_{1,2,4}$ as 
followingł:

$$
\begin{array}{lr}
\phi_{1}=B P P_{1} ; \quad \phi_{2}=B P P_{2} ; \quad \phi=\left(\phi_{1}+\phi_{2}\right) / 2 & \text { plasma potential [V] } \\
V_{f l 2}=L P_{2} ; \quad V_{f l 4}=L P_{4} & \text { floating potential [V] } \\
T_{e}=\left(\phi-V_{f l 2}\right) / 2.2 & \text { electron temperature }[\mathrm{eV}] \\
T_{e 1}=\left(\phi_{1}-V_{f l 2}\right) / 2.2, T_{e 2}=\left(\phi_{2}-V_{f l 2}\right) / 2.2, & T_{e 3}=\left(\phi_{2}-V_{f l 4}\right) / 2.2 \\
I_{s a t}=L P_{1} \quad \text { biased at }-150 \mathrm{~V}, & \text { Ion saturation current }[\mathrm{A}] \\
n_{e}=n_{0} \cdot I_{s a t} / \sqrt{T_{e}} & \text { plasma density }\left[\mathrm{m}^{-3}\right]
\end{array}
$$

where the scalar coefficient $n_{0}$ is defined such that $n_{e}$ equals to the absolutely calibrated Lithium beam diagnostic in region around $10 \mathrm{~mm}$ from LCFS. Within this definition, we assume that $T_{e} \approx T_{i}$ and that spatial dimension of $\phi$ turbulent structures are larger than distance between $B P P_{1}$ and $B P P_{2}$, which is later verified in Fig.6a. Eq. (4) is used only for estimation of $T_{\mathrm{e}}$-structure dimensions in Fig.6a,c.

These defined quantities are then statistically processed in common techniques over time window of $2.7 \mathrm{~ms}$. This time window yields a data-set that is large enough for statistics, containing on average 3 to 15 blobs and is much longer than autocorrelation time (see later in Fig.3b). $3 \mathrm{~ms}$ is, however, short enough to treat the probe as steady because it moves less than $2.7 \mathrm{~mm}$ which is much shorter than typical SOL width, $\lambda_{S O L}$. To further ensure statistical homogeneity within the $3 \mathrm{~ms}$ window, frequencies within $0<f<300 \mathrm{~Hz}$ are removed. In addition, persisting frequency peaks from background noise are also identified and removed from the signal.

Examples of time-evolution and corresponding probability distribution functions in middle SOL are shown in Fig.3a,b.

\section{Steady-state radial profiles}

First, at each radial position we compute the lowest statistical moments, ie. mean value, $\nu$ and standard deviation, $\sigma$ to characterize the relative fluctuation level, $\sigma / \nu$

$$
\begin{aligned}
& \nu_{q}=\langle q\rangle=\sum_{i=1}^{N} q_{i} / N \quad \text { mean } \\
& \sigma_{q}=S T D=\sqrt{\sum_{i=1}^{N}\left(q_{i}-\nu_{q}\right)^{2} / N} \quad \text { standard deviation } \\
& \text { for } q=\left[\phi, T_{e}, n_{e}, V_{f l}\right], N=2.7 \mathrm{~ms} \cdot 2 \mathrm{MHz}=5400 \text { samples. }
\end{aligned}
$$

The radial profiles are shown in Fig.3c,d,e as a function of radial distance from separatrix, mapped to midplane. The mean radial profiles in Fig.3d correspond

$\ddagger$ Theoretically, one should define $\phi$ rather as $\phi=(B P P 1+B P P 2) / 2+\alpha_{b p p} T_{e}$. This is, however, practically problematic since $T_{e}$ is not measured really at positions of the BPP's and the $T_{e}$-field has somewhat fine spatial structure (see Fig.6a). Therefore, Eq. (1) is used instead. 


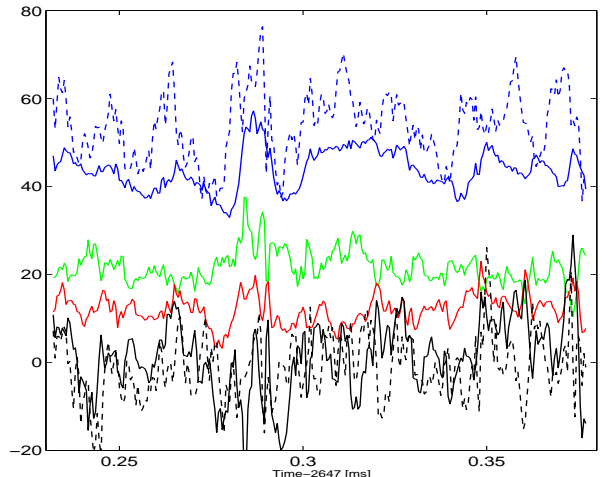

a)

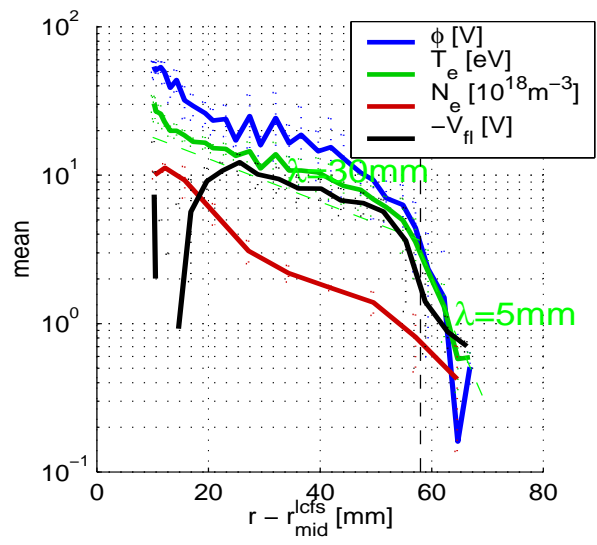

d)

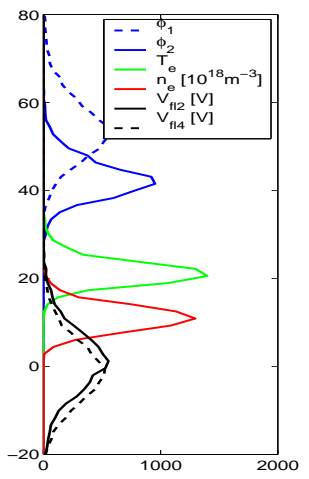

b)

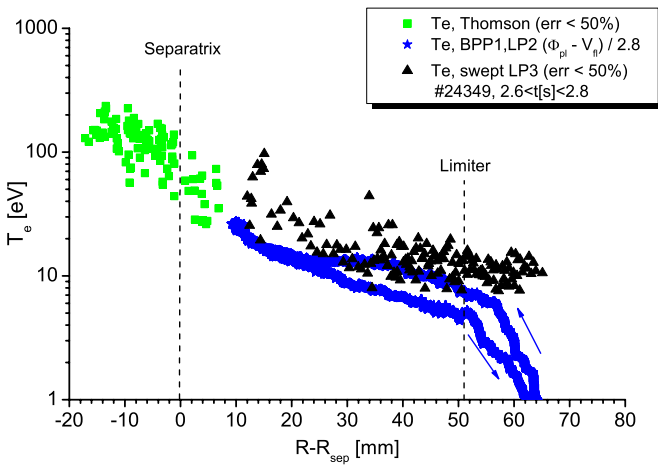

c)

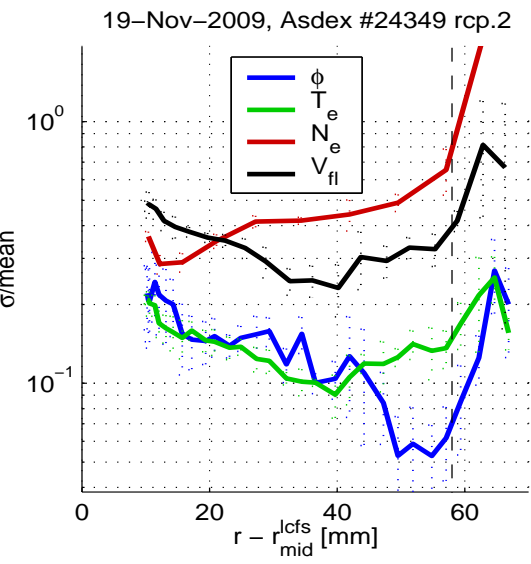

e)

Figure 3. a) example of time-evolution of raw data with b) corresponding probability distribution function. c) $T_{\mathrm{e}}$ is compared with Thomson scattering and slowly-swept Langmuir probe, courtesy of [6]. Radial profiles of time-averaged mean d) (note reversed sign in $V_{\mathrm{f}}$ ) and relative fluctuation level e). Region beyond $58 \mathrm{~mm}$ corresponds to wall shadow where connection length sharply drops by order of magnitude. Standard deviation of fluctuating data are shown by the dotted error-bars.

well to our expectations for a low density plasma discharge. All quantities decrease exponentially,

$$
q=q_{0} \exp (-r / \lambda)
$$

At Fig.3c, $T_{\mathrm{e}}$ derived from Eq. (3) is compared with both Thomson scattering and slowly-swept Langmuir probe $L P_{3}$, showing only those data with credibility higher than $50 \%$. The small hysteresis in the $T_{\mathrm{e}}$ - $\mathrm{BPP}$ is due to plasma cooling down between probe reciprocations in and out.

It is worth noting the discrepancy in the wall-shadow region, where the swept probe measures $T_{\mathrm{e}} \approx 12 \mathrm{eV}$, whilst the $T_{\mathrm{e}}$-BPP drops down to $1 \mathrm{eV}$. We believe that here it is rather the swept probe that yields erroneous values because of the following reason. Parallel damping (of both particles and energy) is inversely proportional to connection 
length, which is strongly reduced in wall shadow,

$$
L_{c}^{S O L} / L_{c}^{\text {wall-shadow }} \approx 10,
$$

where the connection length is computed precisely from magnetic reconstruction as average over both directions to the material surface. Scale-lengths (slope, defined by Eq. (10)) of $\phi, T_{e}$ correspond approximately to $\lambda_{S O L}=30 \mathrm{~mm}$ and $\lambda_{\text {wall }}=5 \mathrm{~mm}$, shown in Fig.3d to emphasize the change in slope between the SOL and wall shadow region. Ratio of those values correspond approximately to ratio of collisionalities, given mainly by Eq. (11),

$$
\frac{\lambda_{S O L}}{\lambda_{\text {wall }}}=\frac{30 \mathrm{~mm}}{5 \mathrm{~mm}} \approx \frac{\nu_{S O L}^{*}}{\nu_{\text {wall }}^{*}}=\frac{50 \pm 20}{10 \pm 3},
$$

where the collisionality $\nu^{*} \propto L_{c} n_{e} T_{e}^{-2}$. The fact that width of radial profile increases with collisionality was observed already in [8]. Therefore, in Fig.3c behaviour of temperature measured by BPP is more reasonable than that from the sweeping probe.

In Fig.3e, fluctuation level of density is strong and increases with distance from LCFS, as observed by many others $[9,10,11,12]$. The fluctuation levels of $T_{\mathrm{e}}$ and $\phi$ are comparable, $\sigma_{T} /\left\langle T_{\mathrm{e}}\right\rangle \approx \sigma_{\phi} /\langle\phi\rangle \sim 0.1$. Notably, the fluctuations are significantly weaker than that of density, consistent with generally observed [12] relation $\sigma_{T} /\left\langle T_{\mathrm{e}}\right\rangle=(0.3 \dot{-}$ $0.4) \cdot \sigma_{n e} /\left\langle n_{\mathrm{e}}\right\rangle$, even though smaller than values reported using sweeping techniques eg. [1]. Overall, the results in Fig.3 are consistent with other published results, well compiled eg. in [12]. This demonstrates correctness of the experimental method, at least that time-averaged data obtained by the Ball-pen probe are reasonable.

\section{Time and spatial characteristics}

This paper is meant to provide experimental base for later comparison of this data with ESEL turbulence model [9]. In principle, such comparison is twofold. First, if agreement is found, we gain strong insight into the physics processes behind the turbulence. Second, the model enlarges the zero-dimensional experimental data into two-dimensional space. This requires experimental information of spatial dimensions which was missing in previous studies $[13,8]$.

\subsection{Experiment}

The experimental technique used to determine the characteristic length in space (spatial correlation, $\lambda_{\text {pol }}$ ) and time (autocorrelation, $\tau_{\mathrm{c}}$ ) is very simple. We compute correlation between two identical quantities at given spatial or temporal distance (ie. shifted). Then, we assume that the correlation is a well-defined and decreasing function of temporal and spatial distance. For simplicity and symmetry, exponential function was chosen:

$$
C\left(q_{1}, q_{2}\right) \stackrel{\text { def }}{=} \frac{\left\langle\tilde{q}_{1} \tilde{q}_{2}\right\rangle}{\sqrt{\left\langle\tilde{q}_{1}^{2}\right\rangle\left\langle\tilde{q}_{2}^{2}\right\rangle}}=\exp \left(-z / \lambda_{\text {pol }}^{q}-t / \tau_{\mathrm{c}}^{q}\right), \quad \text { where } \tilde{q}=q-\langle q\rangle
$$




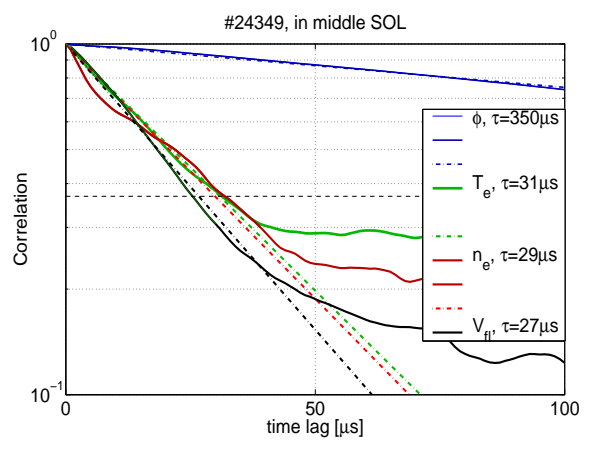

a)

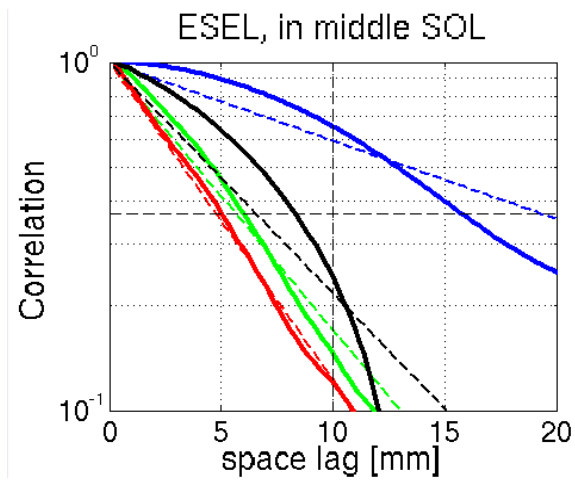

b)

Figure 4. Verification of assumption in Eq. (13), exponential decrease of correlation: a) in time using ASDEX experimental data, b) in poloidal space using ESEL model data. Both time and space lag at $C=e^{-1}=0.37$ correspond to the scale-length $\tau_{\mathrm{c}}^{q}$ and $\lambda_{p o l}^{q}$, respectively.

In $[10,11], e^{-t / \tau_{c}} \cos \left(t / \tau_{c}\right)$ was used which yields fit of similar quality with resulting $\tau_{c}$ systematically higher by $\approx 20 \%$. Since $\tau_{c}$ is found to span across two orders of magnitude (see later in Fig.6b), the exact choice of the fit is unimportant. Validity of exponential decrease in time is demonstrated in Fig.4a using the ASDEX experimental data in the middle of SOL. As for the spatial dependence, many poloidally separated probes are necessary for verification of this relationship, that is generally difficult in experiment and impossible with our probe on ASDEX Upgrade, where we have only one couple of probes yielding a single value for the spatial correlation length. We used therefore the ESEL simulation data to demonstrate this relationship in Fig.4b. The exponential fit describes the behaviour reasonably well with respect to span of $\tau_{\mathrm{c}}^{q}$ and $\lambda_{\text {pol }}^{q}$ across the SOL and among those four quantities.

\subsection{Theoretical expectations}

We base our expectations of structure sizes on the ESEL model. For illustration, an example of turbulence structures is shown in Fig.5. In general, a blob is generated in the velocity shear layer just inside LCFS and then expelled into SOL, accelerated by the interchange instability driven by radial gradient in both pressure and toroidal magnetic field $[9,17]$. A blob is a coherent structure of a size around a centimeter, consisting from two lobes, each with positive and negative potential and vorticity but with maximum density and temperature in its centre. We expect therefore several relationships and facts concerning size of the structures:

- Both temperature and density structures are similar, frozen inside a blob and thus comparable in size $\Rightarrow \lambda_{T}^{\text {pol }} \sim \lambda_{n}^{\text {pol }}$.

- Size of vorticity $\Omega$ structures is somewhat finer since a blob contains both positive and negative lobes, whilst only a single positive peak of $T_{\mathrm{e}} \Rightarrow \lambda_{T}^{\text {pol }} \approx 2 \lambda_{\Omega}^{\text {pol }}$. 


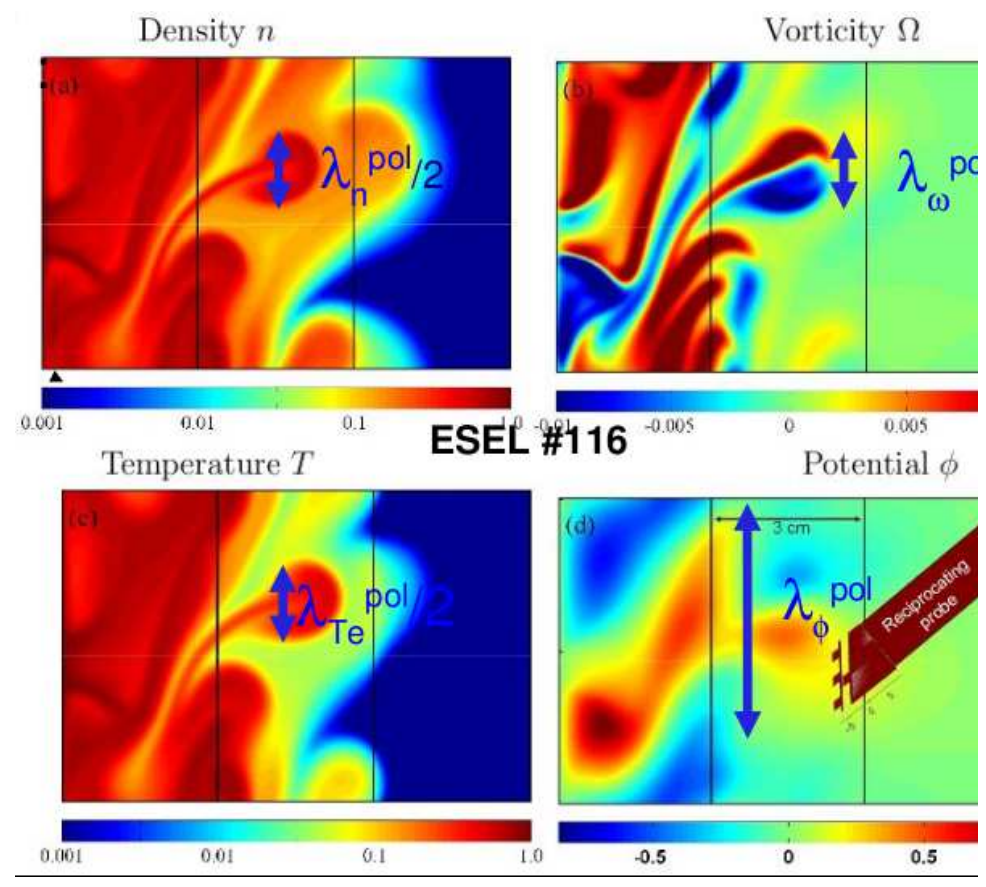

Figure 5. Illustration of how turbulence fields in density, vorticity, temperature and potential look like, generated by the ESEL model. This demonstrates validity of relations Eq. (14) between those structures.

- Since plasma potential is given as $\nabla^{2} \phi=\Omega$ [13, Appendix A], its spatial variation is much smoother than that of $\Omega \Rightarrow \lambda_{\Omega}^{p o l}<<\lambda_{\phi}^{\text {pol }}$.

Therefore, we expect the following relationship:

$$
\lambda_{T}^{p o l} \approx \lambda_{n}^{p o l} \approx 2 \lambda_{\Omega}^{p o l}<<\lambda_{\phi}^{p o l}
$$

Concerning time and velocity of the turbulence field, we expect that all four quantities are frozen into the turbulence field. Therefore, they should all move at the same speed $v=\sqrt{v_{\text {pol }}^{2}+v_{\text {rad }}^{2}}$, where $v_{\text {pol }}$ is determined by the $E_{r} \times B$-drift as $v_{\text {pol }}=-\mathrm{d} \phi / B \mathrm{~d} r$. Since lifetime of the structures is much longer than $\tau_{\mathrm{c}}$, the total velocity is simply given as $\oint v=\lambda / \tau_{\mathrm{c}}$. Since the measurement of $\lambda$ is available only in the poloidal direction, whilst $\tau_{c}$ is determined by speed of plasma flow in its direction, we use $v=A_{r} \lambda_{p o l} \tau_{\mathrm{c}}$, where the aspect ratio $A_{r}$ of blobs were found $[14,15]$ around 2 near LCFS and 1 in far SOL. Therefore, this method yields reliably the total velocity of plasma cross-field flux, up to factor of 2. Finally, we expect the following relationship:

$$
(1-2) \lambda_{\text {pol }} / \tau_{\mathrm{c}} \approx v_{\text {pol }}^{\phi} \approx v_{\text {pol }}^{T} \approx v_{\text {pol }}^{n} \approx-\frac{\mathrm{d} \phi}{B \mathrm{~d} r}
$$

$\S$ Note that direction (sign) of $v$ cannot be resolved by this method. 
Fast measurement of plasma potential, temperature and density in SOL of ASDEX Upgrade9

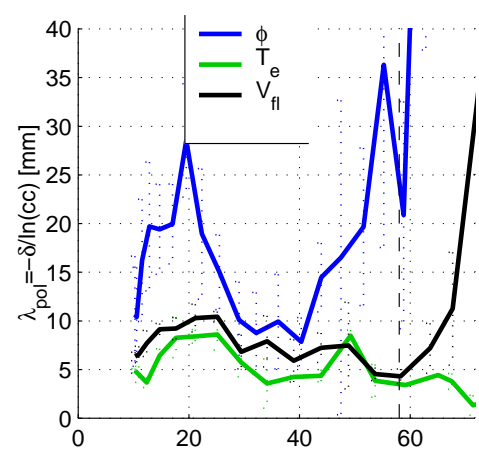

a)

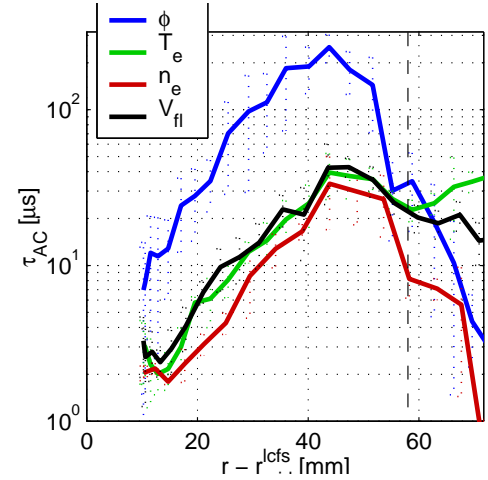

b)

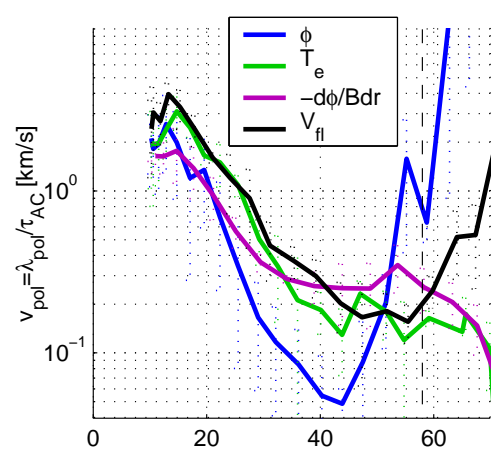

c)

Figure 6. Data-analysis based on Eq. (13) yields radial profiles of a) poloidal spatial dimension $\lambda_{\text {pol }}$ (where $\delta=5$ or $10 \mathrm{~mm}$ ), b) auto-correlation time $\tau_{\mathrm{c}}$ and c) poloidal velocity, $v_{\text {pol }}$. Note that $\lambda_{p o l}$ and $v_{\text {pol }}$ is not available for $N_{e}$ because only a single probe measured $N_{e}$.

\subsection{Comparison}

Never mind that in the wall shadow $\left(r-r_{m i d}^{l c f s}>58 \mathrm{~mm}\right)$ credibility of the data decreases as the signal becomes comparable to the noise, those expectations Eq. (14) and Eq. (15) are successfully verified in SOL in Fig.6 using the experimental data. Notably, $\tau_{\mathrm{c}}$ of potential is much longer than that of other quantities because its spatial scale is much larger but its velocity field is the same. Since many experimentalists measure usually $V_{\mathrm{fl}}$, we show this quantity too, demonstrating that its interpretation is difficult. Note especially that both structure sizes and time-scales of $V_{\mathrm{ff}}$ are closer to that of $T_{\mathrm{e}}$ than $\phi$, in opposite to common expectations. This demonstrates that for computing radial particle and energy fluxes, using floating Ball-pen instead of a Langmuir probe might yield different (and more credible) results.

\section{Conditional average}

Here we use conditional averaging technique to obtain a 'typical' behaviour of all measured quantities as the blob passes across the probe head. We extract and sum together all events when a triggering condition have a well-defined positive peak at time when a blob passes across. Even though good candidate for triggering can be also $T_{\mathrm{e}}$, we chose density because of two reasons: a) it is measured directly whilst $T_{\mathrm{e}}$ is derived non-locally, b) skewness of density $(0.5<S<2.5)$ is much higher:

$$
\left\langle I_{\text {sat }}>2.5 \sigma_{\text {Isat }}+\left\langle I_{\text {sat }}\right\rangle \mid q\right\rangle \stackrel{\text { def }}{=} \Sigma_{i=1}^{B} q\left(-20<t_{i}-d t[\mu \mathrm{s}]<+40\right)
$$

where again $q=\left[\phi, T_{e}, n_{e}, V_{f l}\right]$. Frequency of appearance of those events increases from $f=3 \mathrm{kHz}$ near LCFS up to $f=5 \mathrm{kHz}$ in mid-SOL and drops down to $f=1 \mathrm{kHz}$ in wall shadow; the corresponding number of blobs $B=2.7 \mathrm{~ms} \cdot f$. This we interpret such that near LCFS blobs are borned, whilst in the wall shadow region they tend to fuse 
together into larger objects. The conditionally-averaged signals are shown in Fig.7 for three different positions for all quantities.

\subsection{Plasma potential}

Both plasma potential measurements correspond well to expected blob movement from the $B P P_{1}=\phi_{1}$ upwards to the $B P P_{2}=\phi_{2}$. At $9 \mathrm{~mm}$ from LCFS, the delay of $5 \mu \mathrm{s}$ between peaks in $\phi_{1}$ and $\phi_{2}$ corresponds to poloidal velocity of $10 \mathrm{~mm} / 5 \mu \mathrm{s}=2 \mathrm{~km} / \mathrm{s}$, consistent with result in Fig.6c. The DC difference $\left\langle\phi_{1}\right\rangle-\left\langle\phi_{2}\right\rangle$ comes from the fact that the probe head is inclined by $12^{\mathrm{O}}$ with respect to magnetic surface, resulting in $2 \mathrm{~mm}$ radial displacement between the BPP's. Within $2 \mathrm{~mm}$, the $\langle\phi\rangle$ drops by a value equal to $2 m m \mathrm{~d} \phi / \mathrm{d} r$ computed from the radial profile in Fig.3d, and analogically for $V_{\mathrm{fl}}$.

\subsection{Density}

Density perturbation is short and strong, it reaches amplitude up to $250 \%$ of the local mean value and drops down with time-scale much shorter than that of temperature. This fact comes, however, mainly from the fact that $I_{\text {sat }}$, dominated mostly by $n_{\mathrm{e}}$ perturbation, is used for triggering the conditional averaging. Therefore, structure in all other quantities are statistically more smoothed out that in density. It has been reported in many papers (eg. $[13,11]$ ) that $I_{\text {sat }}$ conditional waveform is asymmetric, it rises faster than it drops, as a result of a blob passing radially outwards, leaving behind a trailing wake. It is obvious from this figure that this time-asymmetry is likely not due to density but rather due to temperature.

\subsection{Electron temperature}

The electron temperature, $T_{\mathrm{e}}$ seems to evolve in only partially expected way. First, temperature increases when density increases. This is expected from any model (eg. [9] or [17]) of high-pressure blobs propagating from LCFS outwards. In experiment, even though fast simultaneous measurements of $T_{\mathrm{e}}$ and $n_{\mathrm{e}}$ are rare, positive correlation is generally observed. Second, the perturbation persists much longer, up to $35 \mu \mathrm{s}$. The perturbation is quite time-asymmetric, it appears rather after the event. This may be an indication that the cross-field transport of heat inside a blob is stronger than transport of particles. Very surprising is, however, observation that at the very centre of the blob, at $d t \approx 0$, the electron temperature drops down for the short time when density is at its maximum. Since $T_{\mathrm{e}}=\left(\phi-V_{\mathrm{fl}}\right) / \alpha$ in Eq. (3), this is clearly a result of sudden increase in $V_{\mathrm{fl}}$ signal at $d t=0$. Attempt to explain this as a result of the erroneous spatially-shifted measurement of $\phi$ cannot explain this: even if we shift the BPP signals (ie. accounting for finite poloidal velocity) by $10 \mathrm{~mm} / \mathrm{v}_{\text {pol }}$, it is the positive $V_{\mathrm{fl}}$ peak which generates negative $T_{\mathrm{e}}$ peak through Eq. (3). Since the floating potential measurement of a Langmuir probe is quite strongly believed to be reliable, this small drop of temperature inside a blob seems real, showing its finite 
structure. Consequently, measured correlation between density and temperature in the middle of SOL is negative, $C\left(n_{\mathrm{e}}, T_{\mathrm{e}}\right) \approx-0.4 \pm 0.2<0$. Note that this observation is still consistent with the generally published positive correlation between density and temperature, because within typical time-scale of fast sweeping (or harmonic) techniques around $10 \mu \mathrm{s}$, positive correlation would be also found for our data. Our surprising negative correlation is a consequence of unusually high time-resolution. On the other hand, far from LCFS, at $33 \mathrm{~mm}$ in the Fig.7, $T_{\mathrm{e}}$ drops by $2 \mathrm{eV}$ at $-5<d t[\mu \mathrm{s}]<5$ even below the time-averaged value. This cannot be explained otherwise than as an experimental error.

We tried to explain this as parasitic influence of pin $L P_{2}$ lying nearby magnetic field line of pin $L P_{1}$ which at the $d t \approx 0$ drives large current. Therefore, for comparison, we plot also signal of pin $L P_{4}\left(V_{f l 4}\right)$ in Fig.3a,b and Fig.7. We verified also that everywhere in SOL all the statistical characteristics and time-scales are identical: $\left\langle V_{f l 2}\right\rangle=\left\langle V_{f l 4}\right\rangle, \sigma_{V f l 2}=\sigma_{V f l 4}, \tau_{c 2}=\tau_{c 4}$. In addition, the "strange" positive peak at $d t \approx 0$ at $L P_{2}$ is well reproduced on $L P_{4}$, keeping in mind that $L P_{2}$ is shifted from $L P_{4}$ poloidally by $10 \mathrm{~mm}$ and radially by $2 \mathrm{~mm}$. Therefore, we conclude that $L P_{2}$ is measuring correctly, namely it is not influenced by the presence of the biased pin $L P_{1}$.

Such anti-correlated temperature with density on short time-scales has been, however, just recently observed in reversed pinch RFX-mod [16]. This suggests that the common picture of blobs containing high density and temperature is true except in the very centre of a blob.

\section{Conclusions}

In this paper, we demonstrated usefulness of the Ball-pen probe, measuring directly plasma potential. In combination with two other Langmuir probes it yields fast and local measurement of the main plasma parameters: density, electron temperature and plasma potential. We found that relative fluctuation level of both temperature and potential is significantly lower than density. Concerning spatial and temporal characteristics of turbulence structures, we found them consistent with general expectations based on a model of interchange turbulence. Conditional averaging of bursty events is also consistent, except from surprising drop of electron temperature at the very centre of a blob.

\section{Acknowledgement}

This work was carried out within the EURATOM Associations IPP.CR, IPP, Ris $\varnothing$ DTU and ÖAW. It was supported by of the Czech Academy of Sciences projects: GA AV KJB100430901 and GA AV 202/09/1467"Multi-range tomographic system for transport studies in tokamak plasmas". This work was also supported by projects P19901 of the Austrian Science Fund (FWF). 

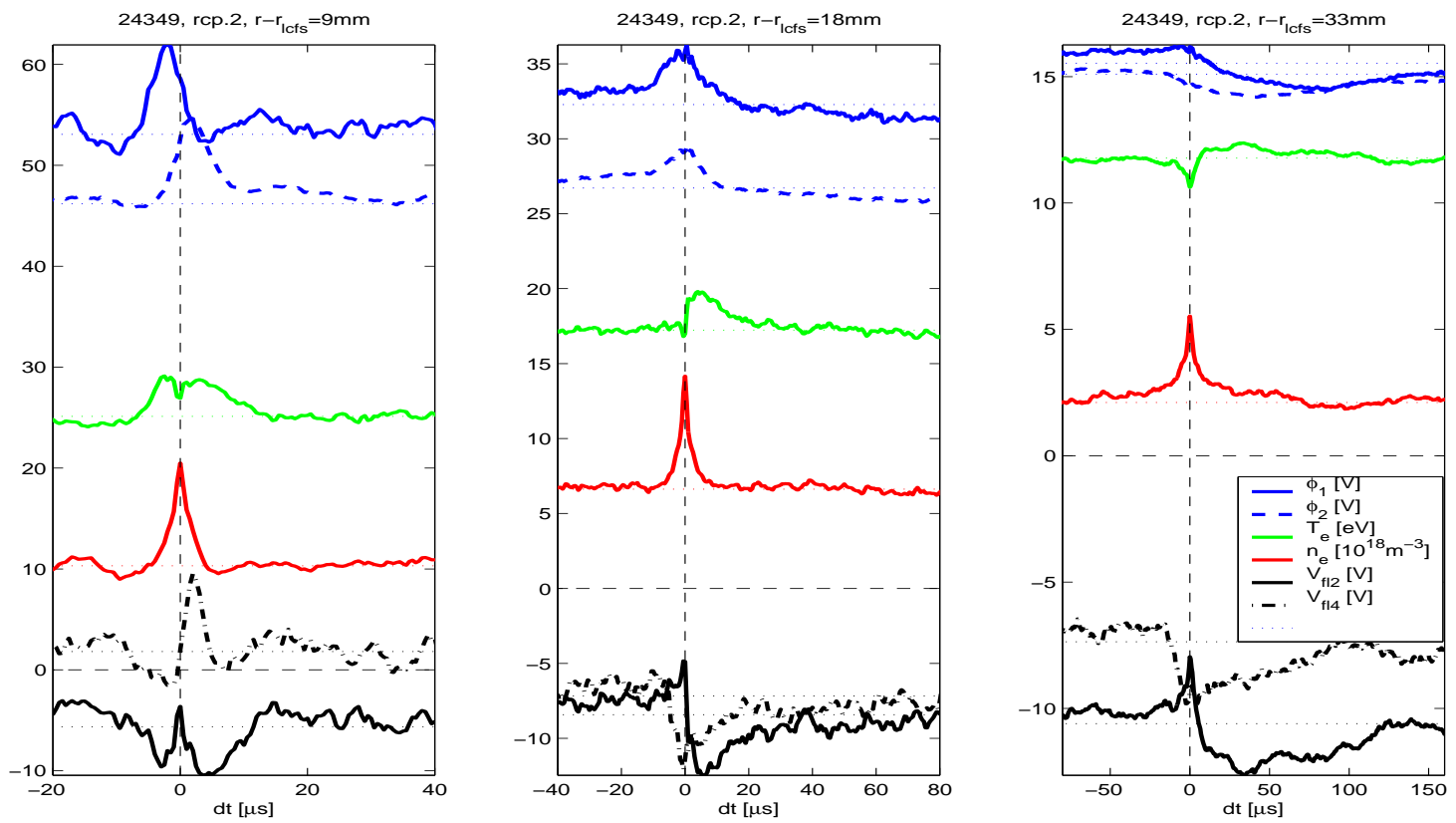

Figure 7. Conditionally-averaged blob passing across the probe head, triggered by peak in $I_{\text {sat }} \propto n_{\mathrm{e}}$ signal. Three positions 9,18,33 $\mathrm{mm}$ from LCFS are shown within time-scale proportional to the local autocorrelation time. Local mean values are shown by the dotted lines.

\section{References}

[1] J.A. Boedo et al Review of Scientific Instruments 70 (1999) 2997

[2] J.P. Gunn et al Czechoslovak J. Phys. 55 (2005), 255

[3] J. Adamek et al Czechoslovak J. Phys. 55 (2005), 235

[4] C. Silva et al Rev. Sci. Instrum. 75 (2004) 4314

[5] J. Adamek et al 36th EPS Conference on Plasma Physics, Sofia, Bulgaria, 2009

[6] J. Adamek, J. Horacek, H W Muller et al Contrib. Plasma Phys. (2010)

[7] H.W. Muller, J. Adamek, J. Horacek et al Contrib. Plasma Phys. (2010)

[8] O.E. Garcia et al Plasma Phys. Control. Fusion 49 (2007) B47

[9] O.E. Garcia et al Phys. Plasmas 12 (2005), 062309

[10] J. Graves et al Plasma Phys. Control. Fusion 47 (2005) L1L9

[11] J. Horacek, R.A. Pitts, J. Graves. Czech. J. Phys. 55 (2005) 271-283

[12] J.A. Boedo, Journal of Nuclear Materials 390391 (2009) 2937

[13] J. Horacek, PhD Thesis No 3524, 2006, EPFL Switzerland, LRP 817/06, ISSN 0458-5895

[14] A.H. Nielsen et al Poster 13th European Fusion Theory Conference 12-15 October 2009, Riga, Latvia.

[15] G.S. Xu et al Nucl. Fusion 49 (2009) 092002. doi:10.1088/0029-5515/49/9/092002

[16] M. Spolaore et al Physical Review Letters 102 (2009) 165001

[17] S.I. Krasheninnikov. Physics Letters A 283 (2001) 368370 